\title{
Aluminium, vecteur énergétique ${ }^{1}$ ?
}

\author{
Patrick Déry, B.Sc., M.Sc., physicien spécialiste en énergétique \\ Groupe de recherches écologiques de La Baie (GREB)
}

La sécurité de l'approvisionnement futur en pétrole se révèle de plus en plus incertaine en raison de problèmes liés à l'investissement, à l'explosion de la demande ou aux conséquences de l'atteinte d'un éventuel « pic pétrolier ${ }^{2}$ Selon AIE, ces problèmes pourraient survenir d'ici 2011. ${ }^{3}$ Or, plus de 95\% du transport mondial repose sur le pétrole. Au Québec, cette proportion est de $99 \%$.

Dans un tel contexte, nos moyens de transport devront être réorganisés et nos déplacements réduits substantiellement. S'il s'avère que les tenants du pic pétrolier ont raison, le défi sera d'autant plus grand que nous devrons composer avec un déclin de la production pétrolière de $2 \%$ à $3 \%$ annuellement, voire plus. La réorganisation en sera donc complexifiée.

Une des pistes de solution consiste à trouver un ou des substituts à ce pétrole dans le secteur des transports. Mais l'usage des biocarburants comme l'éthanol et le biodiesel est de plus en plus contesté car ces productions entraînent une augmentation des prix de la nourriture et une hausse de la pollution agricole. ${ }^{4}$ De plus, ces carburants souffrent d'une faible énergie nette. ${ }^{5}$

L'électrification du transport constitue aussi une avenue possible dans le contexte québécois où la production électrique, grâce à l'énergie hydraulique, est presque à $100 \%$ de source renouvelable. Cependant, les batteries actuelles, qui sont nécessaires pour le transport individuel électrifié, sont encore encombrantes et lourdes. C'est là que l'aluminium pourrait offrir une alternative aux batteries en devenant un porteur d'énergie : l'aluminium est, d'une certaine façon, de l'électricité sous forme solide.

\section{L'aluminium pourrait offrir une alternative aux batteries en devenant un porteur d'énergie}

Actuellement perçu comme un matériau, l'aluminium se transige comme tel sur les marchés mondiaux. Sa consommation, en forte croissance au niveau mondial, particulièrement en Asie, engendre une pression à la hausse sur son prix, comme pour toutes les commodités par ailleurs. De plus, sa fabrication, exigeante en énergie, ajoute à la hausse des prix à la fois de l'énergie et de l'aluminium et ce, dans un contexte où il y a peu de marge de manœuvre dans la croissance de la production énergétique.

\section{Il est fort possible que la demande d'aluminium finisse par se stabiliser et peut-être même décroître}

Avec les rumeurs d'une récession aux ÉtatsUnis susceptible de s'étendre à l'échelle du monde, il est fort possible que la demande d'aluminium finisse par se stabiliser et peutêtre même décroître, surtout si le pic pétrolier survenait prochainement. Les prix élevés de l'aluminium ne pourront alors se maintenir très longtemps. Il n'est pas impossible de voir dans le secteur de l'aluminium ce que l'on a vu, ou plutôt vécu, dans le secteur forestier et agricole, c'est-à-dire un écroulement de la demande et des prix sous la barre des coûts de production. Une question apparait alors : peut-on d'ores et déjà anticiper et planifier des transitions? 


\section{Comparaison des prix}

aluminium, pétrole brut et charbon

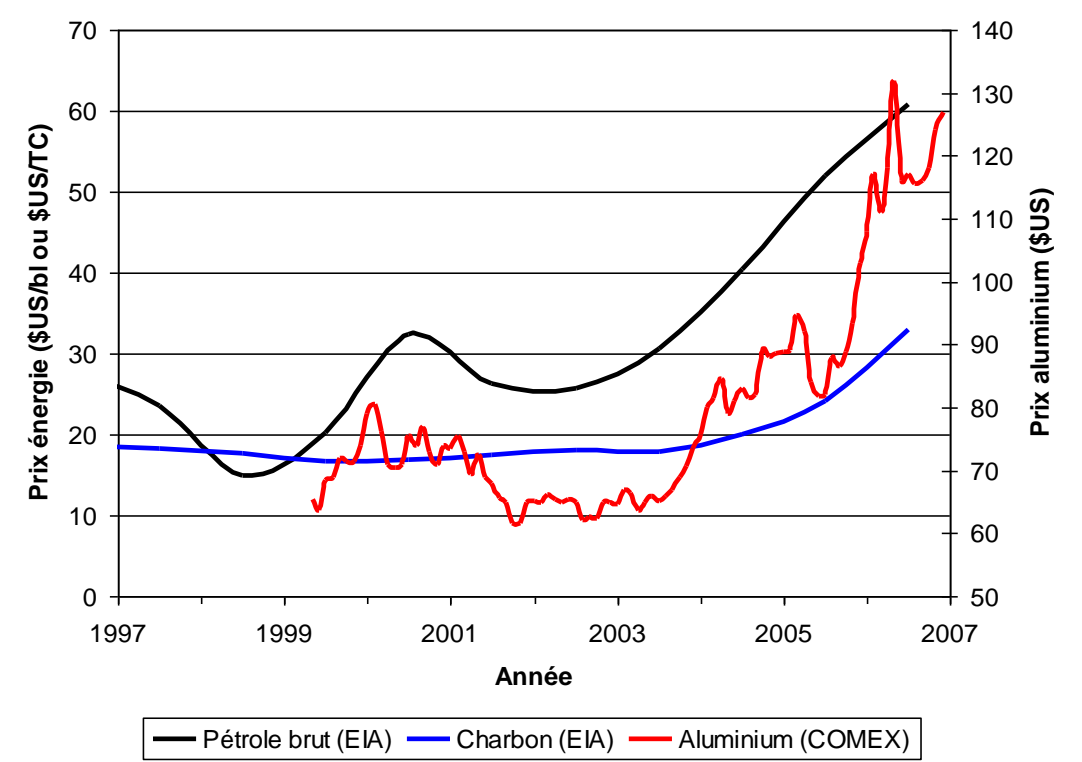

Le marché de l'énergie est un marché en forte croissance et l'atteinte prochaine et probable du maximum de production du pétrole ouvrira le marché des carburants de transport. Aucun substitut viable n'existe actuellement. L'aluminium pourrait alors devenir un candidat intéressant à ce titre.

L'aluminium possède une densité énergétique gravimétrique ${ }^{6}$ de $9,9 \mathrm{kWh} / \mathrm{kg}$. Pour comparer, la densité énergétique gravimétrique du pétrole est de 13,4 $\mathrm{kWh} / \mathrm{kg}$, celle de l'éthanol de $7,8 \mathrm{kWh} / \mathrm{kg}$ et celle des batteries au plomb d'environ 0,05 $\mathrm{kWh} / \mathrm{kg}$.

En plus de posséder une densité énergétique intéressante, l'aluminium comporte d'autres avantages en tant que vecteur énergétique. Il est solide et chimiquement stable dans les conditions normales, il est très abondant dans la nature et bien réparti sur l'ensemble de la planète, il est d'entreposage facile, est aisément recyclable et possède la qualité d'être non-toxique. Au regard de ces faits, l'aluminium est supérieur à l'hydrogène comme vecteur énergétique sur la plupart des points, excepté sur la simplicité d'extraction par électrolyse, l'électrolyse de l'hydrogène étant un processus beaucoup plus facile à réaliser que celle de l'aluminium, surtout à petite échelle.

Il existe trois procédés d'extraction de l'énergie contenue dans l'aluminium, soit la pile à combustible solide (aluminium-air), la pile rechargeable en aluminium et la génération d'hydrogène par oxydation d'aluminium. 


\section{La pile à combustible solide (aluminium-air)}

Cette pile n'est pas rechargeable électriquement mais mécaniquement, comme c'est le cas avec toute pile à combustible. Il faut remplacer ses électrodes par de nouvelles et vidanger l'alumine générée par le processus. Cette alumine peut être recyclée pour refaire de l'aluminium. L'électrolyte doit être stocké à l'extérieur de la pile lorsqu'il n'est pas utilisé, sinon la durée de vie en sera très limitée. La densité énergétique de l'ensemble de la pile se situe à environ 0,25 $\mathrm{kWh} / \mathrm{kg}$ soit 5 fois plus que celle des piles acide-plomb. Toutefois, les coûts de cette pile demeurent encore très élevés et son usage actuel est surtout militaire.

\section{La pile rechargeable en aluminium ${ }^{7}$}

L'annonce en 2004 par Europositron (Finlande) de la résolution des problèmes de corrosion et de non-rechargeabilité de la pile aluminium-air pour la fabrication d'une pile rechargeable demeure à démontrer. Celle-ci ouvre toutefois la possibilité, si cela était concluant, du transport d'électricité par voie maritime ou ferroviaire. ${ }^{8}$ Les sources renouvelables d'électricité, comme le solaire dans les zones désertiques et ensoleillées, par exemple, pourraient rendre la production d'électricité mondiale plus propre et plus disponible tout en diversifiant ses usages comme pour le transport électrifié. Tout cela reste encore bien spéculatif mais suscite certains espoirs. La densité énergétique gravimétrique serait de $1,3 \mathrm{kWh} / \mathrm{kg}$ (25 fois la pile acide-plomb).

\section{La génération d'hydrogène par oxydation d'aluminium9}

L'aluminium, à son point de fusion $\left(660^{\circ} \mathrm{C}\right)$, est hautement réactif à l'eau. À la température ambiante, l'oxydation de la surface génère une gangue protectrice d'alumine qui protège l'aluminium et empêche l'oxydation continue. Toutefois, sous certaines conditions, cette couche protectrice n'est pas formée et l'aluminium poursuit son oxydation. Cette oxydation génère de l'hydrogène pouvant servir comme carburant.

\section{$2 \mathrm{Al}$ (aluminium) $+3 \mathrm{H}_{2} \mathrm{O}($ eau $) \rightarrow 3 \mathrm{H}_{2}$ (hydrogène) $+\mathrm{Al}_{2} \mathrm{O}_{3}$ (alumine) + chaleur}

Les conditions de cette réaction à des températures inférieures au point de fusion sont nombreuses. Il est possible de réaliser des alliages avec certains métaux comme le gallium pour abaisser le point d'oxydation de l'aluminium. On peut aussi réaliser des films de métaux liquides empêchant la formation d'une gangue d'alumine et permettant d'extraire l'énergie contenue dans l'aluminium. Les principaux problèmes d'une économie basée sur l'hydrogène étant le stockage et le transport de celui-ci, l'aluminium pourrait alors résoudre ces deux problèmes à la fois 
Après les crises forestières et agricoles, l'économie de la région du Saguenay-LacSaint-Jean est maintenant très dépendante de sa production d'aluminium. En plus de l'être fortement des carburants fossiles. Comme vecteur énergétique de notre production d'électricité, l'aluminium pourrait remplacer le pétrole dans les transports. À la différence du pétrole, l'aluminium est recyclable et, au Québec, fabriqué à partir de sources renouvelables. De plus, il serait peut-être possible d'utiliser des sources intermittentes comme l'éolien nordique pour sa fabrication. Cela reste à explorer mais l'avantage serait de pouvoir augmenter la limite de la production d'électricité éolienne liée aux réserves des barrages hydroélectriques (le couplage hydro- éolien est limité à une valeur située entre 10 à $20 \%)^{10}$.

La région doit pouvoir se donner les moyens d'anticiper les crises et de trouver des solutions novatrices. Il ne faudrait surtout pas que les mêmes erreurs que dans les secteurs de l'agriculture et de la forêt se répètent.

Le contexte actuel est encore propice à l'investissement dans la recherche de nouvelles utilisations de l'aluminium.

\section{Le contexte actuel est encore propice à l'investissement dans la recherche de nouvelles utilisations de l'aluminium}

\section{Notes et références}

1 Vecteur énergétique : forme intermédiaire en laquelle est transformée l'énergie d'une source primaire pour son transport ou son stockage avant son utilisation. Exemple : électricité, hydrogène...

2 Robert L. Hirsch, Peaking of world oil production: Recent forecasts, DOE NETL. April 2007. National Petroleum Council (US), Facing The Hard Truths About Energy, July 2007.

Government Accountability Office, CRUDE OIL: Uncertainty about Future Oil Supply Makes It Important to Develop a Strategy for Addressing a Peak and Decline in Oil Production, February 2007.

World Energy Council, Survey of Energy Resources 2007, september 2007.

Energy Watch Group, Uranium resources and nuclear energy, December 2006 EWG-Series No 1/2006

Energy Watch Group, Coal: Resources and Future Production, April, 2007.

Energy Watch Group, Crude Oil: The Supply Outlook, October 2007.

Robelius, F. 2007. Giant Oil Fields -The Highway to Oil. Giant Oil Fields and their Importance

for Future Oil Production. Acta Universitatis Upsaliensis. Digital Comprehensive Summaries

of Uppsala Dissertations from the Faculty of Science and Technology . 168 pp. Uppsala.

Darley, Julian, High noon for natural gas: the new energy crisis, Chelsea Green, 2004.

3 Agence internationale de l'énergie, Medium Term Oil Market Report, July 2007.

4 EcoNexus, Biofuelwatch, Carbon Trade Watch (Transnational Institute), Corporate Europe Observatory, Ecologistas en Acción, Ecoropa, Grupo de Reflexión Rural, Munlochy Vigil, NOAH (Friends of the Earth Denmark), Rettet Den Regenwald, Watch Indonesia, Agrofuels - Towards a reality check in nine key areas, June 2007.

Brent D. Yacobucci Randy Schnepf, Ethanol and Biofuels: Agriculture, Infrastructure, and Market Constraints Related to Expanded Production, March 16, 2007, Congressional Research Service report for Congress

Amani Elobeid, Simla Tokgoz, Dermot J. Hayes, Bruce A. Babcock, and Chad E. Hart, The Long-Run Impact of Corn-Based Ethanol on the Grain, Oilseed, and Livestock Sectors: A Preliminary Assessment,

Center for Agricultural and Rural Development, Iowa State University,November 2006.

5 Patrick Déry, Quel rendement pour notre investissement énergétique?, $3{ }^{\text {ième }}$ volet sur l'énergétique régionale au Saguenay-Lac-Saint-Jean, Conseil régional de l'environnement et du développement durable, Groupe de recherches écologiques de La Baie, février 2008.

6 La densité énergétique gravimétrique est la quantité d'énergie que peut libérer un composant de stockage énergétique en fonction de son poids (densité gravimétrique) ou de son volume (densité volumique). En transport, la première est plus importante que la seconde.

7 www.europositron.com

8 Pieter Van Pelt, Cheap Electricity from Iceland, zpenergy, 2004

9 Woodall, Jerry M., The science and technology of aluminum-gallium alloys as a material for energy storage, transport and splitting water, Keynote Address, ECHI-2, April 12 $2^{\text {th }}, 2007$.

10 Gaëtan Lafrance, Mémoire d'expert, www.mrnf.gouv.gc.ca, décembre 2004. 